\title{
Recubrimientos protectores para componentes de turbinas de aviación y de generación de energía depositados por proyección por plasma
}

\author{
A. AGÜERO BRUNA, J. ALVAREZ ALBA, F.J. GARCíA DE BLAS VILLANUEVA Y P. VALLES GONZÁlEZ \\ Instituto Nacional de Técnica Aeroespacial, Área de Materiales Metálicos
}

\begin{abstract}
Los recubrimientos producidos por difusión de aluminio, llamados aluminuros, se emplean para aumentar la resistencia a la oxidación y a la corrosión, incrementando la vida de componentes fabricados con superaleaciones de níquel y cobalto a temperaturas comprendidas entre 900 y $1050^{\circ} \mathrm{C}$. Por ello se emplean muy frecuentemente en turbinas aeronaúticas y de producción de energía y en la industria química. Los aluminuros se obtienen industrialmente mediante cementación o CVD y recientemente se ha demostrado que su resistencia aumenta significativamente cuando se añade cromo (cromoaluminuros). Durante el presente trabajo, se ha realizado un estudio de viabilidad para determinar si la proyección por plasma puede ser utilizada para la producción de estos recubrimientos sobre álabes de turbina. Así pues, se depositaron capas de aluminio y aluminio/cromo sobre probetas de la superaleción base níquel IN100 que a continuación fueron sometidas a un tratamiento térmico de difusión bajo flujo de argón. La caracterización y análisis de los recubrimientos producidos se llevó a cabo mediante técnicas metalográficas, microscopía electrónica de barrido y espectroscopía de dispersión de energía de rayos X. Se realizaron ensayos de oxidación cíclica a $1050^{\circ} \mathrm{C}$ y de corrosión por sulfatos fundidos a $900^{\circ} \mathrm{C}$. Los resultados de este estudio preliminar han sido muy satisfactorios e indican que la proyección por plasma puede ser desarrollada como procedimiento industrial para la producción de recubrimientos aluminuros y cromoaluminuros.
\end{abstract}

Palabras Clave: recubrimientos, proyección por plasma, alta temperatura, corrosión, oxidación, turbinas, aluminuros, cromoaluminuros

Protective Coatings for Aeronautic and Power Generation Turbines Components Deposited by Plasma Spray

Coatings produced by aluminium diffusion, called aluminide are employed to increase the oxidation and corrosion resistance, increasing the life of $\mathrm{Ni}$ and $\mathrm{Co}$ base superalloys components at temperatures comprised between 900 and $1050^{\circ} \mathrm{C}$. Consequently these coatings are frequently employed in aeronautic and power generation turbines as well as in the chemical industry. Aluminides are industrially produced by pack cementation or CVD and recently it has been demonstrated that its resistance significantly increases when $\mathrm{Cr}$ is added (chrome-aluminides). During this work, a feasibility study has been carried out in order to determine if plasma spray can be employed for depositing this type of coatings on turbine blades. Therefore, aluminium and aluminium/chromium layers were deposited on Ni base IN100 superalloy specimens that were subsequently subjected to a diffusion heat treatment under Ar flow. Characterisation and analysis of the coatings were carried out by metallography, SEM and EDS. Cyclic oxidation tests were carried out at $1050^{\circ} \mathrm{C}$ while molten sulphate hot corrosion was performed at $900^{\circ} \mathrm{C}$. The results of this preliminary study are promising and indicate that plasma spray can be developed as an industrial process for production of aluminide and chrome-aluminide coatings.

Key Words: coatings, plasma spray, corrosion, oxidation, high temperature, aluminide, chrome-aluminide

\section{INTRODUCCIÓN}

Los recubrimientos producidos por difusión de aluminio, llamados aluminuros, se emplean para aumentar la resistencia a la oxidación y a la corrosión a alta temperatura de las superaleaciones de níquel y cobalto. A temperaturas comprendidas entre 900 y $1050^{\circ} \mathrm{C}$, estos recubrimientos incrementan la vida de componentes fabricados con estas superaleaciones y por ello se emplean muy frecuentemente en turbinas aeronaúticas, de producción de energía y en la industria química (1).

Existen varios métodos industriales para la deposición de aluminuros. De entre ellos, la cementación ("pack cementation") es hasta el presente el más frecuentemente utilizado debido a su simplicidad como método y a su bajo costo (2). Este proceso consiste en "enterrar" los componentes a recubrir en una mezcla de polvos comprendida esencialmente por aluminio, un haluro de amonio o "activador" y un material inerte de relleno que suele ser $\mathrm{Al}_{2} \mathrm{O}_{3}$. El proceso se lleva a cabo a $700^{\circ} \mathrm{C}-900^{\circ} \mathrm{C}$ ó a $1000^{\circ} \mathrm{C}-1100^{\circ} \mathrm{C}$ bajo atmósfera controlada (generalmente $\mathrm{H}_{2} \mathrm{o} \mathrm{Ar}$ ) y por varias horas. Dependiendo de la temperatura de aplicación se obtienen dos tipos de recubrimientos cuando se emplean superaleaciones de níquel como substrato (3).

- a $700-900^{\circ} \mathrm{C}$ se obtienen aluminuros de alta actividad en los cuales el aluminio difunde predominantemente hacia el interior del substrato formando esencialmente la fase $\mathrm{Ni}_{2} \mathrm{Al}_{3}$

- a $1000-1100^{\circ} \mathrm{C}$ el níquel difunde hacia el exterior generando aluminuros de baja actividad con la fase $\beta-\mathrm{NiAl}$ principalmente 
sobre una zona de interdifusión que contiene precipitados de carburos de metales refractarios (W, Ta, Mo, etc.) y otras fases intermetálicas complejas en una matriz $\mathrm{Ni}_{3} \mathrm{Al}$.

La fase $\mathrm{Ni}_{2} \mathrm{Al}_{3}$ es muy frágil y se requiere por lo tanto someter los componentes con aluminuros de tan alta actividad a un tratamiento térmico a $1050^{\circ} \mathrm{C}$ para obtener recubrimientos con tres zonas: la más extensa es una matriz de la fase $\beta$-NiAl más estable y dúctil, con precipitados y carburos dispersos, la intermedia de $\beta$-NiAl puro y la más interna de interdifusión como en el caso de los aluminuros de baja actividad. La función del recubrimiento es la de generar una capa fina y protectora de $\mathrm{Al}_{2} \mathrm{O}_{3}$ sobre superficie para prevenir la oxidación o la corrosión del material subyacente. Cuando debido a tensiones causadas mayoritariamente por choques térmicos, dicha capa se desprende, o cuando es atacada por sales fundidas procedentes del combustible o del medio ambiente (marino por ejemplo), la capa es regenerada a expensas del aluminio del recubrimiento. Así, un recubrimiento será más duradero mientras más estable sea esta capa de óxido o mientras tenga una mayor y más duradera capacidad de regeneración.

También se ha comprobado que los aluminuros modificados con cromo poseen una mayor resistencia a la corrosión a alta temperatura (4). Estos recubrimientos presentan la misma microestructura de los aluminuros pero con un mayor contenido de cromo en solución sólida en la capa superior y precipitado en la zona de interdifusión. El cromo se puede co-depositar junto con el aluminio por cementación, o con anterioridad a la etapa de deposición de aluminio también mediante cementación. Es de hacer notar que la co-deposición no es todavía un método fiable ya que no es totalmente reproducible (5).

Existen además otros procesos que pueden emplearse para producir aluminuros y que incluyen fundamentalmente cualquier método que permita depositar aluminio, y si el proceso tiene lugar a temperaturas inferiores a $1000^{\circ} \mathrm{C}$, será necesario someter al componente a un tratamiento térmico adecuado para generar la fase $\beta-\mathrm{NiAl}$ con la estructura correspondiente a la de los aluminuros de baja actividad. Entre estos procesos se encuentra la deposición química en fase vapor (CVD), la deposición física en fase vapor (PVD), la electrodeposición, la proyección térmica, aplicación de "slurries", electroforesis, etc. (6).

En particular se destaca la proyección por plasma, que permite la deposición de capas de aluminio a partir de sus polvos y que ha sido seleccionada para este estudio. La proyección por plasma presenta una alternativa algo más cara con respecto a la cementación y a la CVD, pero que se justifica cuando ya se dispone de los equipos adecuados, y para volúmenes de producción no muy elevados como es el caso de álabes de turbina reparados. En el presente trabajo se expondrán los resultados preliminares obtenidos al depositar aluminio y cromo sobre la superaleación base níquel IN100, mediante proyección por plasma atmósferica y luego someter estos recubrimientos a tratamientos térmicos apropiados para generar los correspondientes aluminuros y cromoaluminuros de níquel. De igual forma, se presentan los resultados de ensayos de oxidación y corrosión a alta temperatura de estos recubrimientos junto con los de un cromoaluminuro comercial depositado por cementación.

\section{PROCEDIMIENTO EXPERIMENTAL}

\subsection{Materiales}

Los polvos de Al para la proyección por plasma fueron suministrados por Praxair (pureza: 99,9\%, granulometría:45-106 um) y los de Cr por Sulzer Metco (pureza: 99,9\%, granulometría 45-75 $\mu \mathrm{m})$. La superaleacion IN100 (0,18\% C,
$60,5 \% \mathrm{Ni}, 10 \% \mathrm{Cr}, 15 \% \mathrm{Co}, 3 \% \mathrm{Mo}, 5,5 \% \mathrm{Al}, 0,01 \%$ B, $5 \% \mathrm{Ti})$ se obtuvo de álabes suministrados por el Ejército de Aire (Maestranza de Albacete). El recubrimiento cromoaluminuro comercial también fue suministrado por la Maestranza de Albacete.

\subsection{Deposición de recubrimientos}

Los recubrimientos fueron obtenidos en una unidad a escala industrial controlada por ordenador (SNMI), consistente en una antorcha de plasma DC (TS6) montada en un robot (ABB) con 6 ejes de rotación y conectada a un alimentador de polvos vibratorio y doble. Las probetas de IN100 fueron chorreadas con $\mathrm{Al}_{2} \mathrm{O}_{3}$ (angular, granulometría 350-420 $\mu \mathrm{m}$ ) y desengrasadas con acetona en un baño ultrasónico antes de ser recubiertas.

\subsubsection{ALUMINUROS}

Se llevó a cabo una serie de experimentos sobre probetas planas de IN100 con la finalidad de depositar capas de aluminio uniformes, con porosidad mínima y espesor controlado. Basándose en estudios anteriores, se seleccionaron los principales parámetros que pueden influir sobre la calidad de los recubrimientos:

- Gasto másico

- Intensidad de plasma

$0,5-1,8 \mathrm{Kg} / \mathrm{h}$

- Distancia antorcha-probeta

$475-500 \mathrm{~A}$

- Gases plasmágenos:

$\begin{array}{ll}\mathrm{H}_{2} & 0-51 / \mathrm{min} \\ \mathrm{Ar} & 30-501 / \mathrm{min} \\ \mathrm{He} & 5-201 / \mathrm{min}\end{array}$

- No. de pasadas de antorcha: $1-2$

Todos los experimentos se realizaron bajo presión atmosférica pues no se hizo necesaria la proyección bajo presión reducida para obtener recubrimientos de calidad adecuada.

\subsubsection{CROMOALUMINUROS}

Se co-depositó una mezcla de $\mathrm{Al}$ y $\mathrm{Cr}$ ( $10 \%$ en peso de $\mathrm{Cr}$ ) por proyección por plasma atmosférica colocando dicha mezcla en un solo alimentador de polvos y optimizando los parámetros de deposición indicados en el párrafo 2.2.1 hasta obtener recubrimientos uniformes.

\subsection{Tratamientos térmicos}

El tratamiento térmico al que fueron sometidas las probetas recubiertas fue de 1 hora a $700^{\circ} \mathrm{C}$ y 3 a $1050^{\circ} \mathrm{C}$ bajo flujo de argón.

\subsection{Caracterización}

La microestructura de los recubrimientos se analizó por microscopía electrónica de barrido (MEB) equipado con un sistema de microanálisis por dispersión de energías de Rayos X (EDS).

\subsection{Ensayos}

\subsubsection{OXIDACIÓN CÍCLICA}

En el ensayo de oxidación cíclica se sometieron las probetas a choques térmicos consistentes en un calentamiento rápido (6 min) hasta $1050^{\circ} \mathrm{C}$, mantenimiento durante 50 minutos, enfriamiento forzado hasta $65^{\circ} \mathrm{C}$ en 3 minutos. El grado de oxidación 

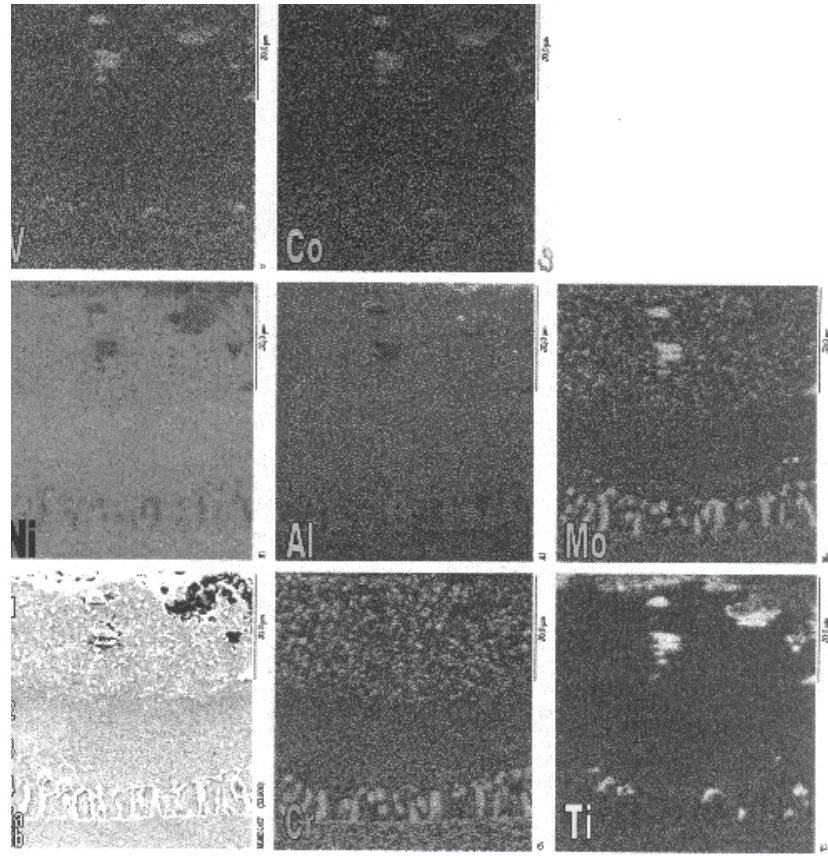

$30 \mathrm{~km}$

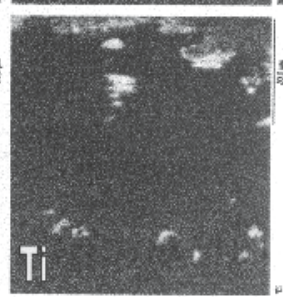

seura I Mapa EDS de lus principules elemsentos del recubrimicnto de al heminuro de níquel producidu per

Figura 1. Mapa EDS de los principales elementos del recubrimiento de aluminuro de níquel producido por proyección por plasma y un tratamiento térmico de difusión.

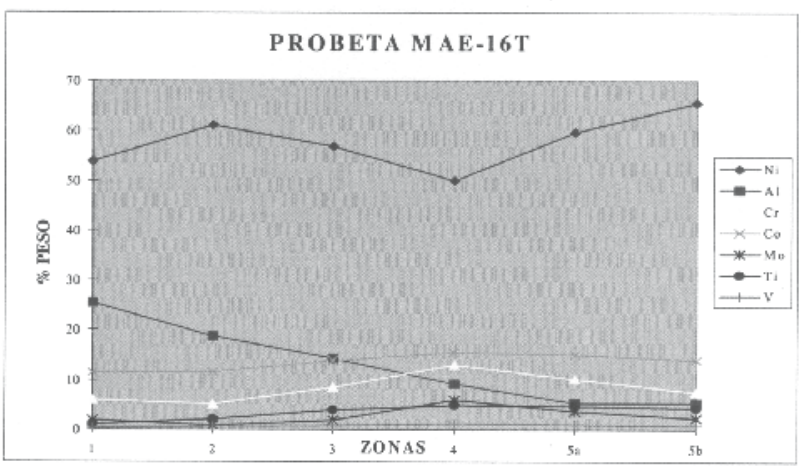

\begin{tabular}{|c|c|c|c|c|c|c|}
\hline ZONAS & $\mathbf{1}$ & $\mathbf{2}$ & $\mathbf{3}$ & $\mathbf{4}$ & $\mathbf{5 a}$ & $\mathbf{5 b}$ \\
\hline $\mathbf{N i}$ & 53,86 & 61,00 & 56,89 & $49,8,5$ & 59,75 & 65,54 \\
\hline $\mathrm{Al}$ & 25,36 & 18,62 & $\mathbf{1 4 , 1 9}$ & 9,16 & 5,24 & 5,29 \\
\hline $\mathbf{C r}$ & 5,79 & 5,05 & 8,45 & 13,05 & 10,22 & 7,53 \\
\hline $\mathbf{C o}$ & 11,28 & 11,61 & $\mathbf{1 3 , 7 5}$ & 15,51 & 14,99 & 13,91 \\
\hline $\mathbf{M} \mathbf{0}$ & 1,92 & 0,79 & 1,85 & 5,92 & 3,63 & 2,20 \\
\hline $\mathrm{Ti}$ & 1,05 & 2,07 & 3,84 & 4,96 & 4,70 & 4,23 \\
\hline $\mathbf{V}$ & 0,48 & 0,54 & 0,85 & 1,21 & 1,04 & 0,87 \\
\hline $\mathbf{M n}$ & 0,15 & 0,24 & 0,11 & 0,29 & 0,29 & 0,29 \\
\hline $\mathbf{F e}$ & 0,10 & 0,08 & 0,07 & 0,05 & 0,13 & 0,13 \\
\hline
\end{tabular}

files de concentración de una probeta recubierta de Al después de haber sido sometida a tı

Figura 2. Perfiles de concentración de una probeta recubierta de $\mathrm{Al}$, después de haber sido sometida a tratamiento térmico (\% en peso). Los números corresponden a las posiciones indicadas en la figura 1.
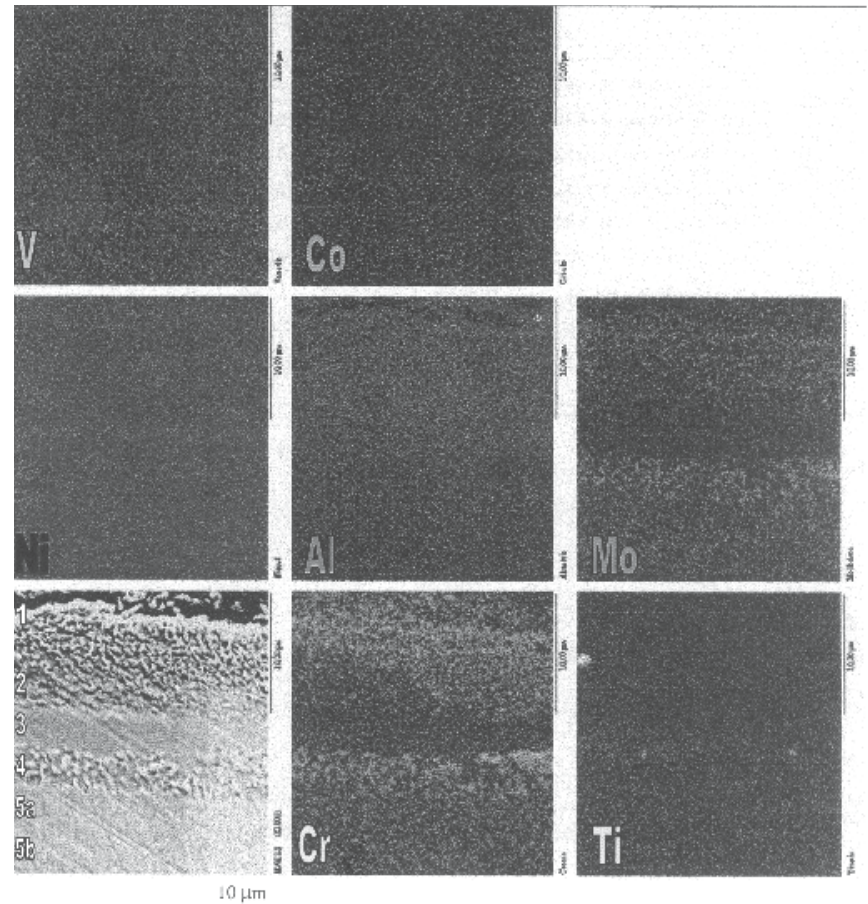

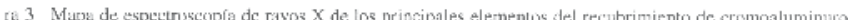

Figura 3. Mapa EDS de rayos X de los principales elementos del recumiento de cromoaluminuro depositado por proyección por plasma y tratamiento térmico de difusión.

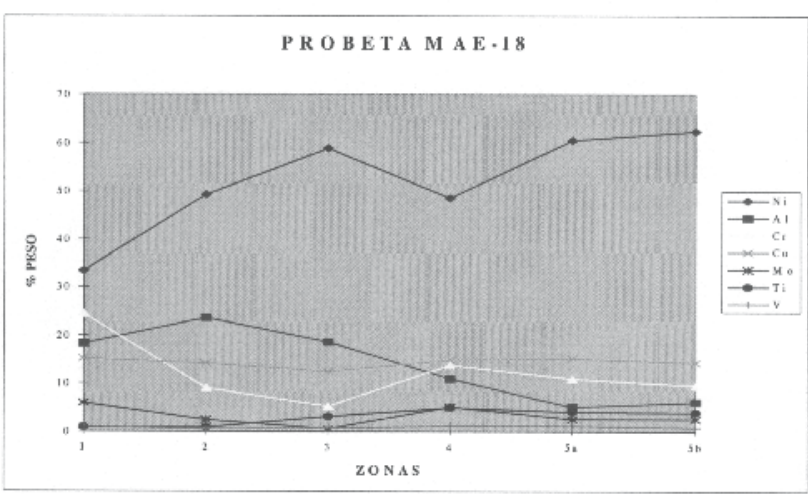

\begin{tabular}{|l|l|l|l|l|l|l|}
\hline ZONAS & $\mathbf{1}$ & $\mathbf{2}$ & $\mathbf{3}$ & $\mathbf{4}$ & $5 \mathrm{a}$ & $\mathbf{5 b}$ \\
\hline $\mathrm{Ni}$ & 33,24 & 49,16 & 58,76 & 48,48 & 60,42 & 62,39 \\
\hline $\mathbf{A l}$ & 18,24 & 23,48 & 18,53 & 10,98 & 5,05 & 6,13 \\
\hline $\mathrm{Cr}$ & 24,35 & 8,97 & 5,26 & 13,83 & 11,00 & 9,48 \\
\hline $\mathrm{Co}$ & 15,24 & 14,20 & 12,54 & 14,89 & 15,12 & 14,35 \\
\hline $\mathrm{Mo}$ & 6,06 & 2,40 & 0,49 & 5,09 & 2,52 & 2,52 \\
\hline $\mathrm{Ti}$ & 0,77 & 0,83 & 3,14 & 4,99 & 4,07 & 3,90 \\
\hline $\mathrm{V}$ & 1,03 & 0,51 & 0,67 & 1,13 & 1,15 & 0,84 \\
\hline $\mathrm{Mn}$ & 0,51 & 0,25 & 0,29 & 0,28 & 0,37 & 0,13 \\
\hline $\mathrm{Fe}$ & 0,56 & 0,19 & 0,32 & 0,33 & 0,32 & 0,26 \\
\hline
\end{tabular}

files de concentración de una probeta recubierta de $\mathrm{Al}-10 \% \mathrm{Cr}$ después de haber sido so

Figura 4. Perfiles de concentración de una probeta recubierta de Al-10\% $\mathrm{Cr}$, después de haber sido sometida a tratamiento térmico (\% en peso). Los números corresponden a las posiciones indicadas en la figura 3. 
se determinó por microscopía óptica de secciones transversales de las probetas tomadas en intervalos regulares de ciclos.

\subsubsection{CORROSIÓN A ALTA TEMPERATURA}

El ensayo se realizó recubriendo las probetas de sulfato de sodio y de magnesio (60-40\% molar) cada $20 \mathrm{~h}$ y exponiéndolas a un caudal de $21 / \mathrm{min}$ de una mezcla de aire y $0,25 \%$ en volumen de $\mathrm{SO}_{2}$ en un horno tubular a $900^{\circ} \mathrm{C}$. A intervalos de $20 \mathrm{~h}$ de ensayo se extrajeron probetas para su análisis metalográfico. Debe tenerse en cuenta que, como en el caso de la oxidación cíclica, estas condiciones de ensayo son muy severas en la forma de reducir la duración de los ensayos, y al mismo tiempo obtener datos comparativos referentes a los distintos recubrimientos evaluados incluyendo un recubrimiento comercial.

\section{DISCUSIÓN DE RESULTADOS}

\subsection{Deposición y Caracterización de recubrimientos}

\subsubsection{ALUMINUROS}

Recubrimientos de aluminio con espesor de $80 \pm 5 \mu \mathrm{m}$, uniformes y con niveles de porosidad aceptables $(<5 \%)$ fueron obtenidos de forma controlada. También se realizó una serie de experimentos para determinar la repetibilidad del proceso la cual se demostró, obteniéndose una variación de espesor promedio de $\pm 5 \mu \mathrm{m}$.

Basándose en experiencias previas en lo que se refiere a tratamientos térmicos de probetas recubiertas con aluminio por otras técnicas, se sometió a las probetas recubiertas en nuestros laboratorios a un tratamiento de $1 \mathrm{~h} \mathrm{a} 700^{\circ} \mathrm{C}$ y $3 \mathrm{~h}$ a $1050^{\circ} \mathrm{C}$ bajo flujo de argón. La figura 1 muestra un corte transversal del recubrimiento resultante de este tratamiento así como los mapas de concentración de los principales elementos obtenidos por MEB-EDS mientras que la figura 2, incluye los perfiles de concentración de dichos elementos. Se observa una estructura correspondiente a un aluminuro de alta actividad con las tres zonas anteriormente descritas y que concuerda con los recubrimientos producidos por cementación (3).

\subsubsection{CROMOALUMINUROS}

A estos recubrimientos se les aplicó el mismo tratamiento que para los aluminuros, y como se observa en la figura 3, se obtuvo una estructura similar a la del aluminuro puro. Sin embargo en el perfil de concentraciones mostrado en la figura 4 se observa una concentración de cromo bastante más elevada en la superficie del recubrimiento y que baja rápidamente para caer a niveles comparables con los del aluminuro puro. Por otro lado, al comparar los mapas de concentración de cromo de los recubrimientos con o sin este elemento (figuras 3 y 1 respectivamente) parece haber una mayor concentración de cromo en la capa correspondiente a la zona externa.

\subsection{Ensayos}

Como se mencionó, en los ensayos de oxidación cíclica y de corrosión por sulfato fundido se incluyeron probetas de un recubrimiento cromoaluminuro comercial con el fin de establecer comparaciones.
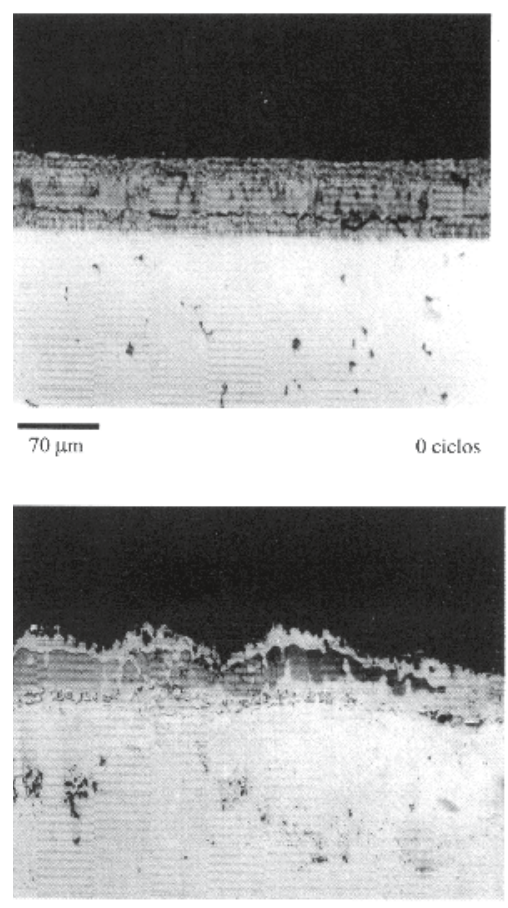

200 ciclos

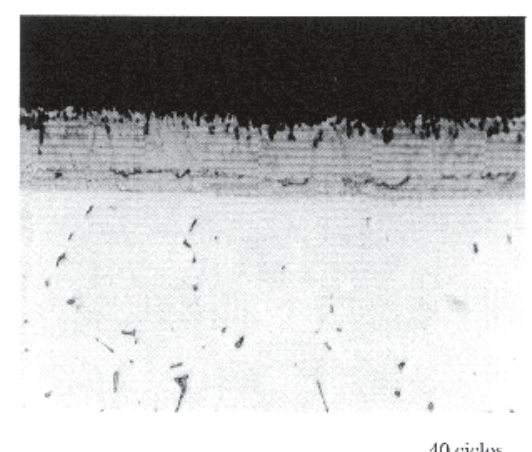

40 ciclos

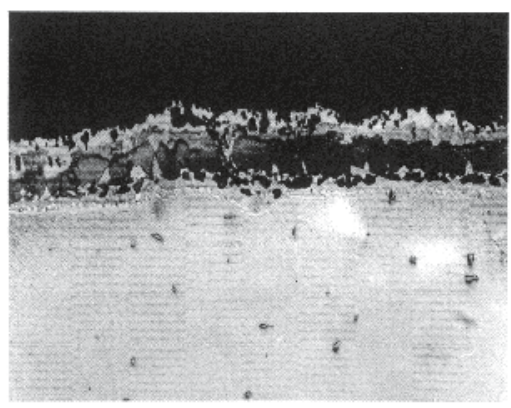

300 ciclos
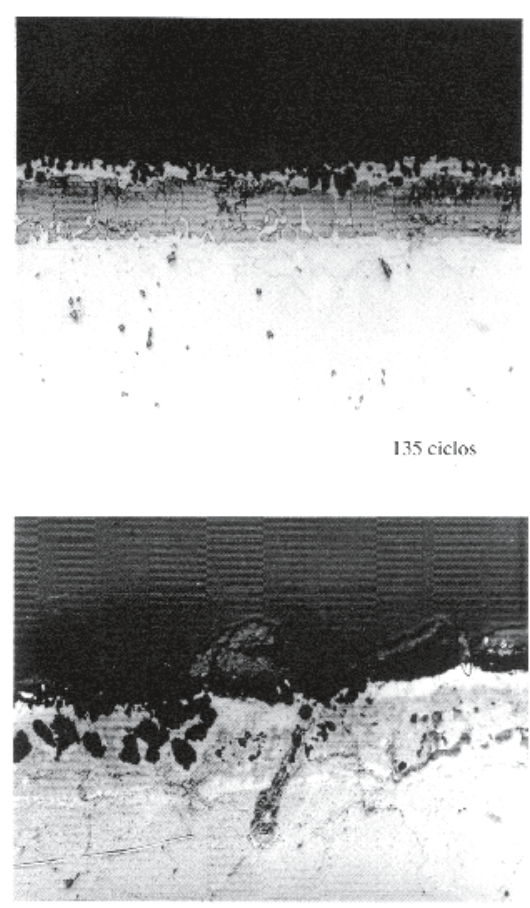

400 ciclos

Fiøura 5 Recubrimiento CrAl comercial después del ensavo de oxidación cíclica a 0. 40. 135. 200) 300) v 400 ciclox

Figura 5. Recubrimiento CrAl comercial después del ensayo de oxidación cíclica a 0,40, 135, 200, 300 y 400 ciclos. 


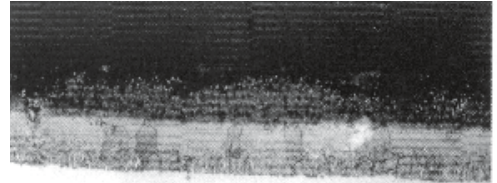

$\overline{70 \mu \mathrm{m}}$
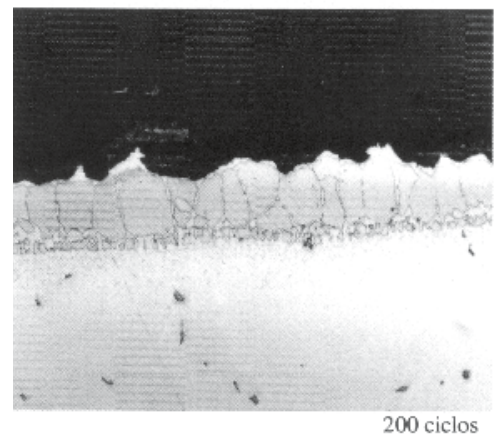
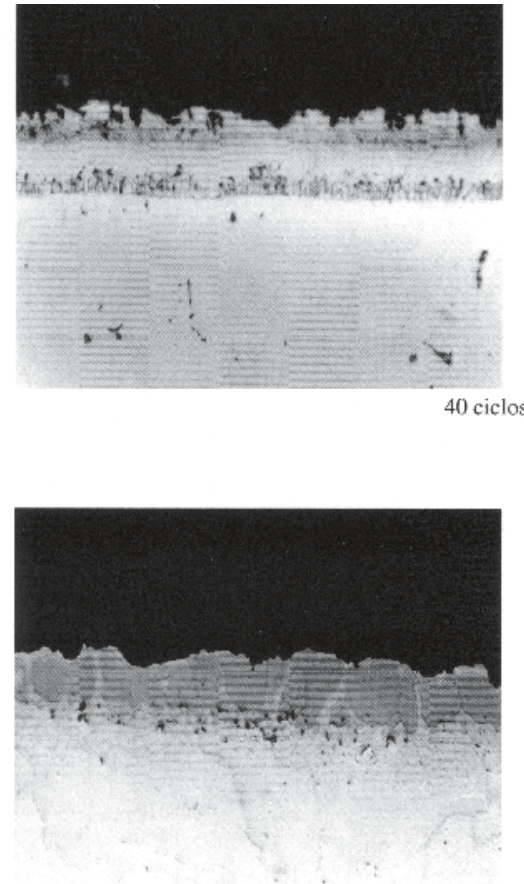

300 ciclos

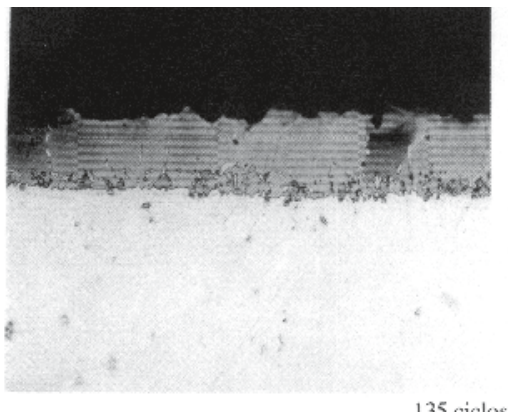

135 ciclos

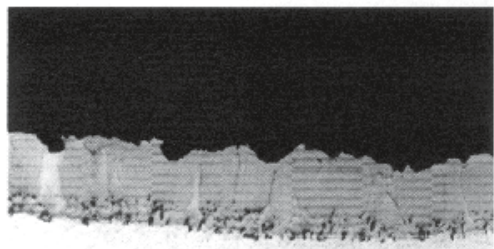

Figura 6 Recubrimiento de Al después del ensayo de oxidación cíclica a 0,40,135, 200,300 y 400 ciclos

Figura 6. Recubrimiento de Al después del ensayo de oxidación cíclica a 0,40, 135, 200, 300 y 400 ciclos.
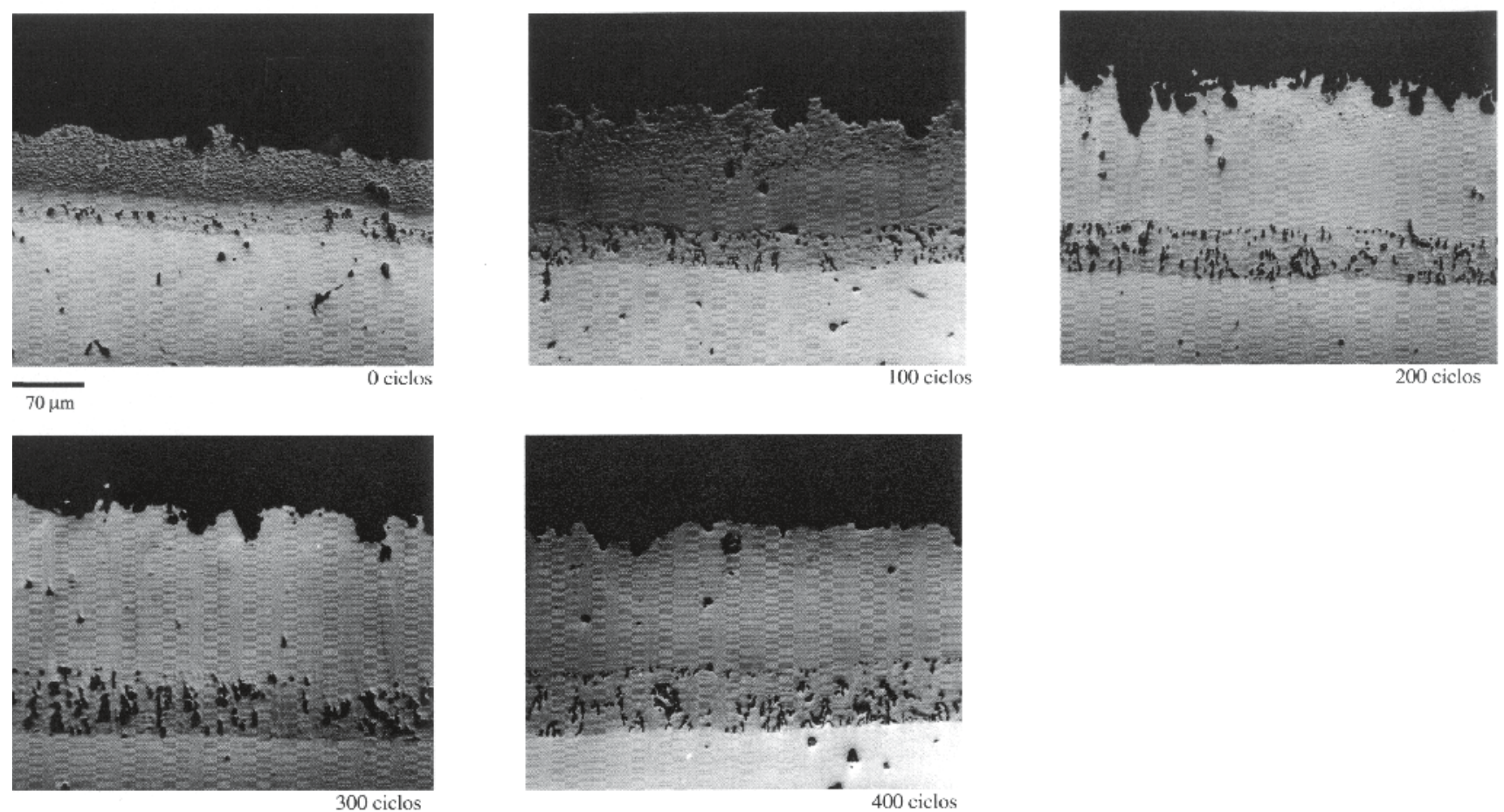

Figura 7. Recubrimiento de Al-10\%Cr después de un ensayo de oxidación a $0,100,200,300$ y 400 ciclos

Figura 7. Recubrimiento de Al-10\%Cr después del ensayo de oxidación a 0,40, 135, 200, 300 y 400 ciclos. 


\subsubsection{OXIDACIÓN CÍCLICA}

\subsubsection{Cromoaluminuro comercial producido por cementación}

La figura 5 muestra los cortes transversales de probetas a 0 , 40, 135, 200, 300 y 400 ciclos de ensayo. A 40 ciclos ya puede verse formación de óxidos en la zona superior del recubrimiento, pero las otras dos zonas permanecen más o menos intactas. A 135 ciclos hay bastante degradación pues la fase $\beta$-NiAl de la zona superior se ha transformado en $\gamma^{\prime}\left(\mathrm{Ni}_{3} \mathrm{Al}\right)$ por pérdida de $\mathrm{Al}$; a 200 ciclos hay todavía mayor grado de degradación con mayor porcentaje de fase g' en todo el recubrimiento y finalmente a 300 ciclos quedan trazas de recubrimiento en el mejor de los casos y mayoritariamente queda la fase $\gamma^{\prime}$ que no es protectora. A 400 ciclos sólo se observa esta última fase sobre toda la superficie de la probeta.

\subsubsection{Aluminuro depositado por proyección por plasma}

La figura 6 ilustra la evolución de la oxidación después de 40, 135, 200, 300 y 400 ciclos y el comportamiento es similar al del recubrimiento comercial. Sin embargo, el recubrimiento producido en nuestro laboratorio parece tener una resistencia ligeramente mayor ya que a 135 ciclos parece haber menor porcentaje de fase $\gamma^{\prime}$ que en el recubrimiento comercial. A 200 ciclos esta diferencia de comportamiento se hace más patente, a 300 ciclos todavía quedan restos de recubrimiento en la mayor parte del álabe mientras que a 400 hay zonas con transformación total en fase $\gamma^{\prime}$ pero quedan restos de fase $\beta$-NiAl. Este comportamiento, algo mejor que el del recubrimiento comercial, puede deberse a que los ensayos se han realizado sobre un número muy reducido de probetas y que las diferen- cias observadas se deben simplemente a variaciones en la calidad del mismo recubrimiento. Estas variaciones están relacionadas con la reproductibilidad y la uniformidad asociadas a la técnica de deposición y se observarían mucho mejor si la evolución de la oxidación se sigue de forma gravimétrica. Por otro lado, es también posible que el recubrimiento depositado por proyección sea mejor que el depositado por cementación y apoyando esta hipótesis se encuentran trabajos publicados en los que se afirma que estos últimos recubrimientos contienen contaminantes provenientes de la mezcla de polvo con la cual está en contacto durante la cementación (7). Estos contaminantes serían perjudiciales para la estabilidad del recubrimiento y se encontrarían ausentes en las capas depositadas por proyección por plasma. Es necesario en consecuencia realizar más experimentos.

\subsubsection{Cromoaluminuro depositado por proyección por plasma}

Este recubrimiento presenta una resistencia significativamente mayor a la oxidación cíclica que los dos anteriores como puede observarse en la figura 7. A medida que aumenta el número de ciclos se observa una transformación progresiva de la microestructura ya que poco a poco la zona más externa va desapareciendo hasta quedar sólo la fase $\beta$-NiAl. A 400 ciclos no hay evidencias de penetración al substrato ni de aumento de la fase $\gamma^{\prime}$. Algunos autores (8) han postulado que el cromo forma una barrera de difusión en la zona de interdifusión del recubrimiento lo cual explicaría el comportamiento observado. Sin embargo, es necesario realizar un análisis más exhaustivo para poder verificar esta hipótesis, y además poder explicar porqué este recubrimiento es mucho más resistente que el producido comercialmente por cementación. Es de hacer notar
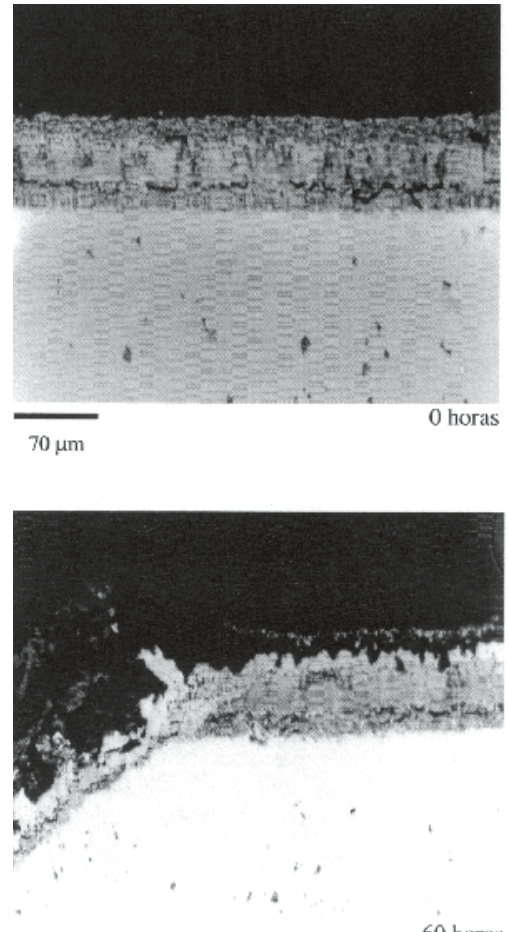

60 horas

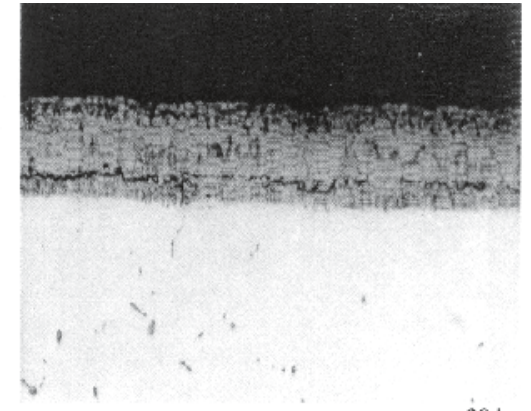

20 horas

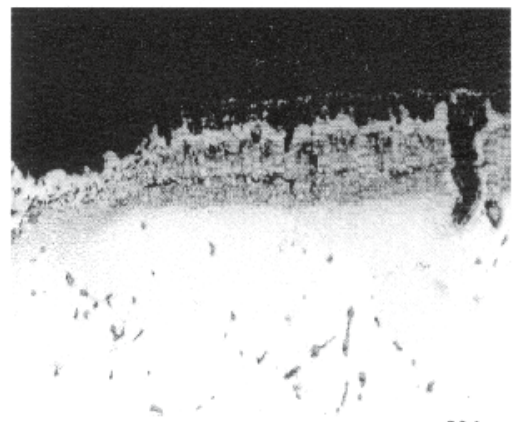

80 horas

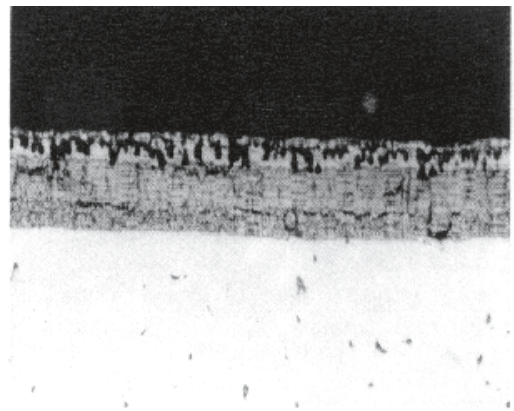

40 horas

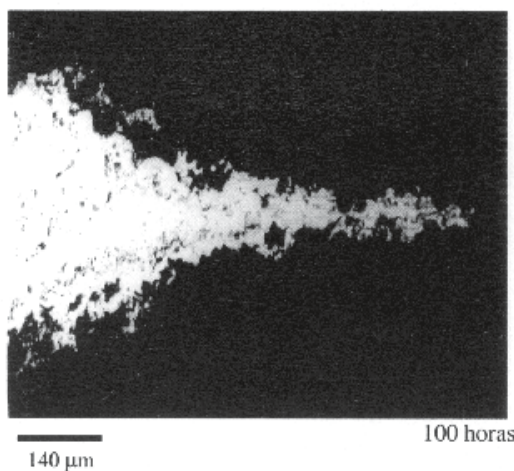

gura 8. Recubrimiento CrAl comercial después del ensayo de corrosión durante $0,20,40,60,80$ y $100 \mathrm{~h}$. La imagen de $100 \mathrm{~h}$ tiene una menor magnificación para poder mostrar el "Figura 8. Recubrimiento de CrAl comercial después del ensayo de corrosión a 0, 20, 40, 60, 80 y 100 h. La imagen de 100 h tiene una menor magnificación para poder mostrar el deterioro sufrido por el borde de salida de un álabe. 

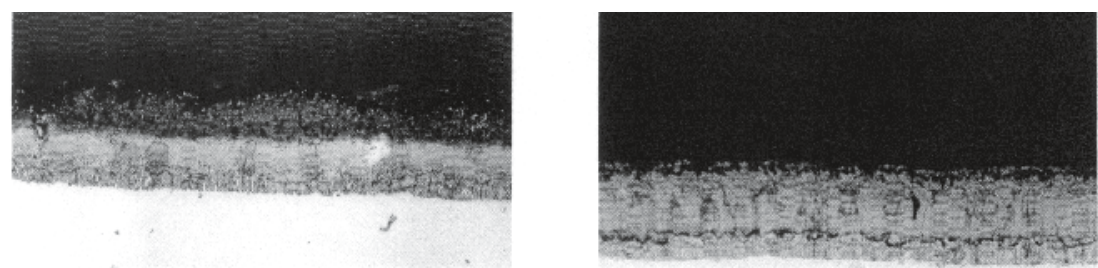

$\overline{70 \mu \mathrm{m}}$

0 horas

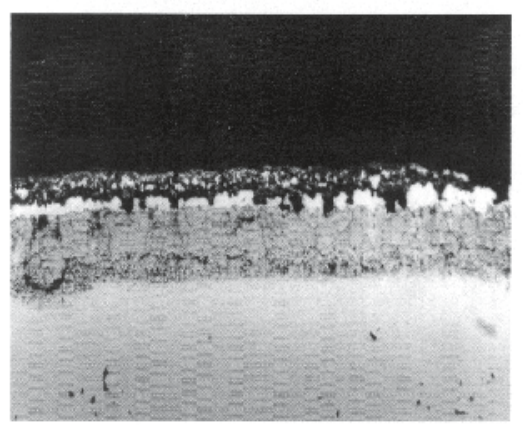

60 horas

20 horas

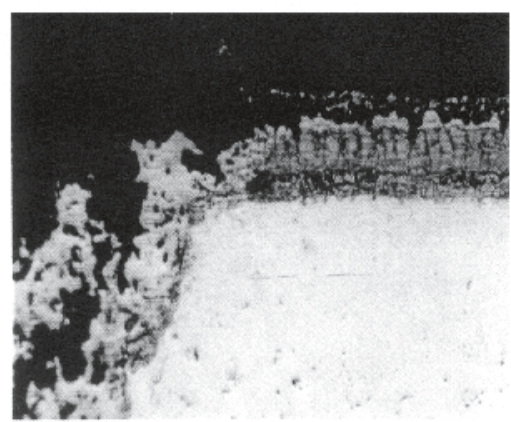

80 horas
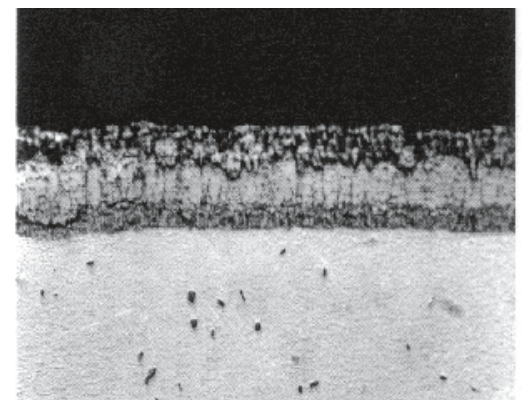

40 horas

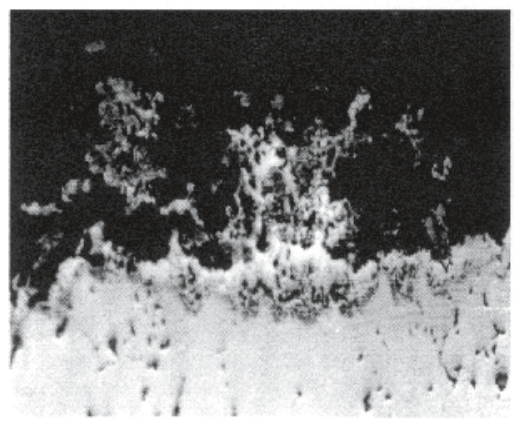

100 horas

Figura 9. Recubrimiento de Al después del ensayo de corrosión durante $0,20,40,60,80$ y 100 horas.

Figura 9. Recubrimiento de Al después del ensayo de corrosión a 0, 20, 40, 60, 80 y 100 horas.
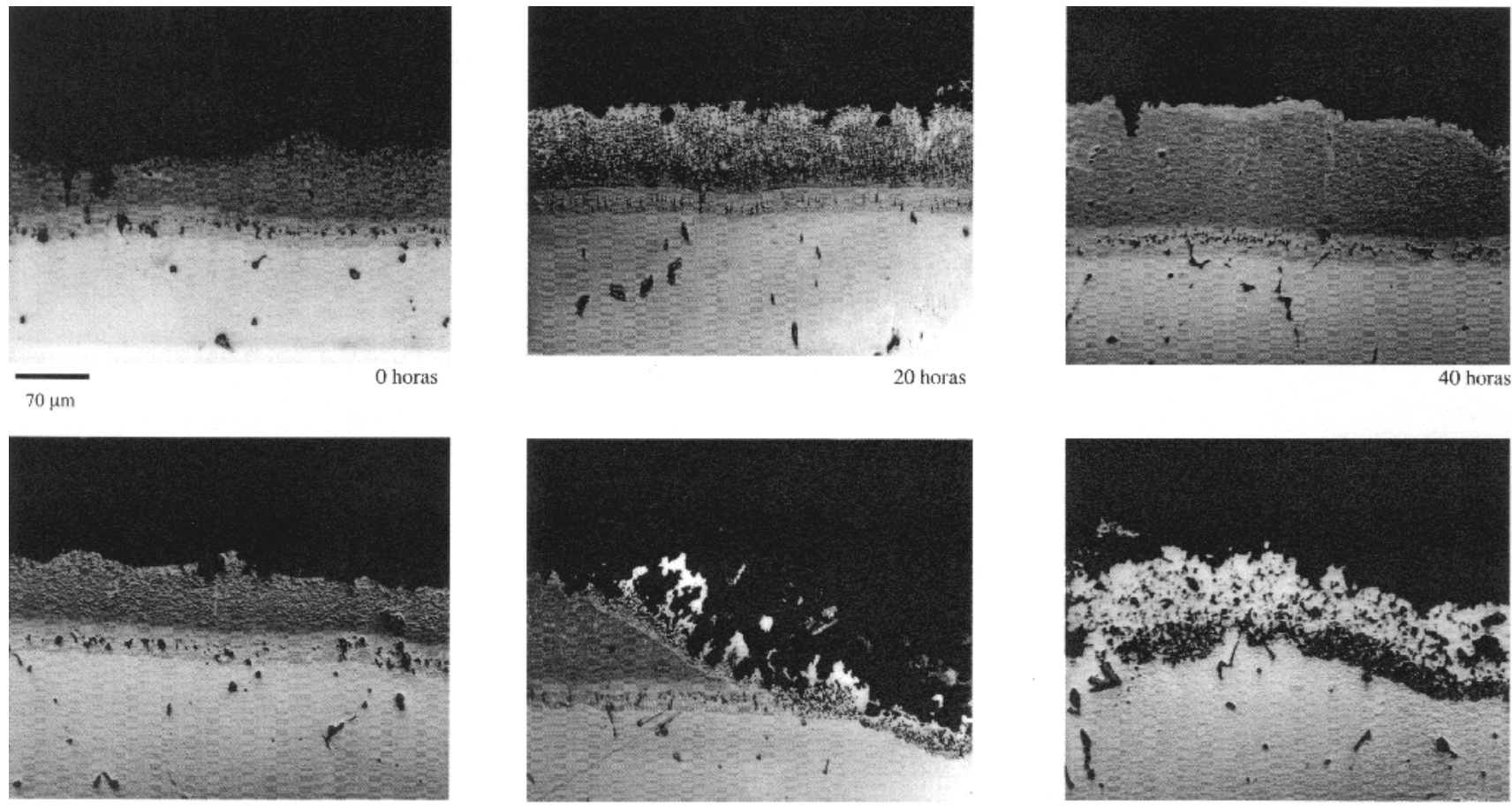

60 horas

80 horas

100 horas

Figura 10. Recubrimiento de Al-10\%Cr después de un ensayo de corrosión a 0, 20, 40, 60, 80 y 100 horas.

Figura 10. Recubrimiento de Al-10\%Cr después de un ensayo de corrosión a 0, 20, 40, 60, 80 y 100 horas. 
que es muy difícil, si no imposible, el poder controlar las cantidades relativas de aluminio y cromo que se depositan por cementación mientras que por proyección por plasma esto resulta muy sencillo.

\subsubsection{CORROSIÓN A ALTA TEMPERATURA}

El mecanismo de corrosión a alta temperatura difiere del de oxidación cíclica en muchos aspectos, pero sobre todo en que consta de un período de incubación lento durante el cual se forma una capa de óxido protectora que en una segunda etapa se pierde para comenzar el ataque, primero del recubrimiento y finalmente del substrato. Esta última etapa de ataque del substrato es muy rápida comparada con las otras dos y se conoce con el nombre de corrosión catastrófica.

\subsubsection{Cromoaluminuro comercial producido por cementación}

La figura 8 muestra los cortes transversales de probetas a 0 , 20, 60, 80 y $100 \mathrm{~h}$ de ensayo. Como puede verse, ya a $20 \mathrm{~h}$ hay evidencia de ataque en la zona superior del recubrimiento que progresa significativamente a las $40 \mathrm{~h}$. A las $60 \mathrm{~h}$ se observan zonas donde el recubrimiento ha desaparecido y hay evidencia de corrosión catastrófica aunque la mayoría de la superficie del álabe todavía tiene recubrimiento. A las 80 horas la situación se invierte pues quedan muy pocas zonas con recubrimiento y en la figura correspondiente, se puede ver también una zona donde la corrosión catastrófica está a punto de comenzar. A 100 $\mathrm{h}$ ya no queda recubrimiento y se puede observar en la figura cómo la probeta ha perdido su forma original.

\subsubsection{Aluminuro depositado por proyección por plasma}

Este recubrimiento presentó un comportamiento muy similar al comercial como se observa en la figura 9.

\subsubsection{Cromoaluminuro depositado por proyección por plasma}

Este recubrimiento también presenta una gran estabilidad frente a la corrosión por sulfatos fundidos como se muestra en la figura 10. Mientras que a las 100 horas de ensayo los otros dos recubrimientos habían desaparecido por completo en este caso todavía queda capa.

\section{CONCLUSIONES}

Los resultados obtenidos indican que es viable depositar recubrimientos aluminuros y cromoaluminuros mediante proyección por plasma, con microestructuras muy similares si no idénticas a las de los obtenidos comercialmente. Por otra parte los recubrimientos cromoaluminuros obtenidos al proyectar mediante plasma una mezcla $\mathrm{Al} 10 \% \mathrm{Cr}$ seguido de tratamiento térmico presentan una mayor estabilidad frente a la oxidación cíclica y a la corrosión por sulfatos fundidos. Habrá que proseguir con una segunda fase en la que se depositarán recubrimientos con diferentes contenidos de cromo y se realizarán ensayos de laboratorio con un número estadísticamente significativo de probetas así como ensayos en banco de pruebas. $\mathrm{Al}$ mismo tiempo se realizarán análisis microestructurales con el objetivo de intentar determinar el efecto del contenido de cromo y las causas de la mayor estabilidad que confiere a los recubrimientos. Finalmente habrá que demostrar que la tecnología funciona a nivel de producción y si lo hace de forma competitiva con las otras técnicas disponibles.

\section{AGRADECIMIENTOS}

Los autores expresan su agradecimiento al Capitán E. Blanco Muñoz por su contribución y apoyo a este trabajo que es parte de un proyecto general de recuperación de álabes de turbina financiado parcialmente por la Maestranza de Área de Albacete y a todo el personal del Área de Materiales Metálicos del INTA, en particular a I. Villar González, R. Muelas Gamo y A. Román Gárate por toda la ayuda prestada.

\section{BIBLIOGRAFÍA}

1. Stringer, "Coatings in the Electricity Supply Industry: Past, Present and Opportunities for the Future", Surf. Coat. Technol., 108-109, 1-9- (1998)

2. G.W. Goward, "Progress in Coatings for Gas Turbine Airfoils", Surf. Coat. Technol., 108-109, 73-79 (1998).

3. G.W. Goward D.H. Boone, "Mechanisms of Formation of Nickel aluminide Coatings on Nickel-Base Superalloys", Oxidation of Metals, 3(5), 475-495 (1971)

4. J.F. Nejedlik, “Development of Improved Coatings for Nickel-and Cobalt-Base Alloys", AFML-TR-70-208 (1970)

5. R. Bianco y R.A. Rapp, “Pack Cementation Aluminide Coatings on Superalloys: Codeposition of Cr and Reactive Elements", J. Electrochem. Soc., 140(4), 11811190 (1993).

6. N.R. Lindblad, "A Review of the Behaviour of Aluminide Coated Superalloys", Oxidation of Metals, 1(1), 143-170 (1961).

7. B.M. Warnes, D.C. Punola, “Clean Diffusion Coatings by Chemical Vapour Deposition", 94-95, 1-6 (1997).

8. E. Fitzer, H.J. Maurer, "Diffusion and Precipitation Phenomena in Aluminized and Chromium-aluminized Iron and Nickel base Alloys", en "Materials and Coatings to resist High Temperature Corrosion", editado por D.R. Holmes y A. Rahmel, Aplied Science Publishers LTD, London, p. 253-270 (1978). 